\title{
Direct MALDI-MS Analysis of Cardiolipin from Rat Organs Sections
}

\author{
Hay-Yan J. Wang, Shelley N. Jackson, and Amina S. Woods \\ National Institute on Drug Abuse-Intramural Research Program, National Institute of Health, Baltimore, \\ Maryland, USA
}

\begin{abstract}
Cardiolipins (CL) are mitochondria specific lipids. They play a critical role in ATP synthesis mediated by oxidative phosphorylation. Abnormal CL distribution is associated with several disease states. MALDI-MS and MALDI-MS/MS were used to demonstrate in situ analysis and characterization of CL from tissue sections of organs containing high concentrations of mitochondria. Once the experimental parameters were established, a survey of CL distribution in heart, liver, kidney, leg muscle, and testis was undertaken. The major CL specie in the heart muscle, leg muscle, liver, and kidney is the (18:2) ${ }_{4} \mathrm{CL}$, while liver and kidney also contain a minor specie, $(18: 2)_{3} /(18: 1)$ CL. The major CL specie in testis is the $(16: 0)_{4}$ CL. The CL species distribution in various organs appeared to be in agreement with prior reports. Overall, proper matrix selection, tissue section handling, instrument tuning, and the inclusion of cesium ion in matrix ensured successful in situ MALDI-MS and MALDI-MS/MS analysis of CL. Upon modification and standardization, this method could be streamlined for rapid pathological diagnosis with short turnaround time in clinical settings. (J Am Soc Mass Spectrom 2007, 18, 567-577) (C) 2007 American Society for Mass Spectrometry
\end{abstract}

$\mathrm{D}$ iphosphatidylglycerol [1,3-bis(sn-3'-phosphatidyl)-sn-glycerol; cardiolipin (CL)] belongs to a unique category of lipid. It consists of two phosphatidic acids linked by a central glycerol. The CL molecule contains four fatty acid chains, three glycerols, and two phosphates [1] (Structure 1). The name "cardiolipin" was given to a lipid from beef heart first purified by Pangborn in 1942 [2]. CL substituents were later quantitatively determined by Macfarlane and colleagues, who subsequently proposed the dimeric structure of this molecule based on their quantitative analysis [3-5].

The two phosphatidic acids in CL connect to the central glycerol at the two terminal positions, leaving the central 2'- hydroxyl group of glycerol unmodified. Each phosphatidyl moiety of CL contains a distinct chiral center. The diphosphatidyl arrangement in CL could give rise to diastereomers and create a unique chemical environment for each phosphate group. The central 2'- hydroxyl group is thought to result in intramolecular hydrogen bonding that alters the $\mathrm{pK}_{2}$ of $\mathrm{CL}$ and to generate the acid-anion in the head group domain of the molecule. This proposal was supported by a titration experiment where the $\mathrm{pK}_{2}$ of regular CL was above 8 , but became equivalent to its $\mathrm{pK}_{1}$ (less than 4.0) when the 2 -hydroxyl group was replaced by a hydrogen [1]. Thus, in spite of the presence of two

Published online December 6, 2006

Address reprint requests to Dr. A. S. Woods, National Institute on Drug Abuse Abuse-Intramural Research Program, National Institute of Health, 333 Cassell Drive, Baltimore, MD 21224, USA. E-mail: awoods@ intra.nida.nih.gov phosphate groups, CL carries only one negative charge under normal physiological conditions.

In mammalian cells, CL is located in the inner membrane of mitochondria, and accounts for 10 to $20 \%$ of total mitochondrial lipids [6]. Hence, a high concentration of CL in organ tissue usually indicates a high amount of mitochondria in such organs whose high metabolic activities are fueled by large amounts of ATP generated from mitochondrial oxidative phosphorylation. In mammals, high amounts of mitochondria can be found in myocardium, liver, kidney, and skeletal muscle. In prokaryote circle, CL is also associated with organisms in the eubacteria subkingdom that utilize oxidative phosphorylation as their energy source.

Much of mitochondrial cytochrome $c$ (Cyt C) is tightly bound to the $\mathrm{CL}$ in its membrane. The Cyt C-CL complex functions as a peroxidase to scavenge excessive $\mathrm{H}_{2} \mathrm{O}_{2}$ generated by oxidative phosphorylation. CL is also an integral part of Complexes III and IV, which are an integral part of the electron transport chain generating electrochemical proton gradients across the inner mitochondrial membrane. In addition, $\mathrm{CL}$ is also the critical component for the assembly of mitochondria supercomplex consisting of Complexes III and IV [7]. Several earlier studies of oxidative stress-mediated apoptosis demonstrated that mitochondria directly or indirectly subjected to oxidative stress disrupted the homeostasis between CL and Cyt C [7-9] and caused the dissociation of Cyt C-CL complexes. Upon dissociation, $\mathrm{Cyt} C$ is released from the inner mitochondrial membrane and eventually escapes to the cytoplasm, 
<smiles>[R10]OCC(COP(=O)([O-])OCC(O)COP(=O)([O-])OCC(CO[R17])C([R20])O[R12])C(=O)O[R17]</smiles>

Structure 1: CL

$\mathrm{M}-\mathrm{H}$ or $\mathrm{M}+\mathrm{Cs}^{+}-\mathbf{2 H}$

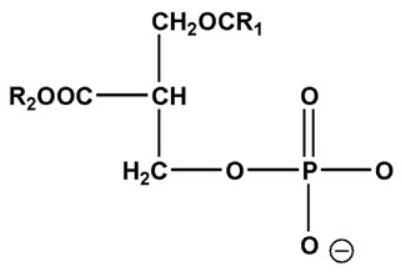

Structure 2:

$\mathbf{a} / \mathbf{b}$ ion

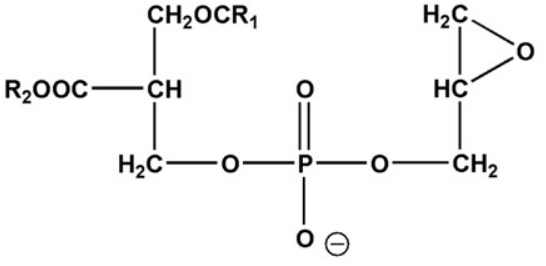

Structure 3:

$[a+56]^{-} /[b+56]^{-}$

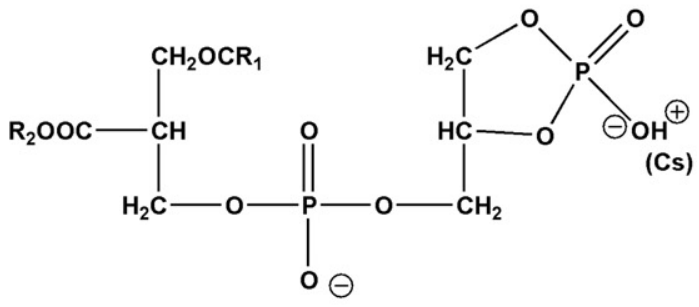

Structure 4:

$[a+136]^{-} /[b+136]^{-}$

or

$\left[\mathrm{a}+136+\mathrm{Cs}^{+}\right]^{-} /\left[\mathrm{b}+136+\mathrm{Cs}^{+}\right]^{-}$ where it triggers a cascade of events leading to programmed cell death [7].

Many pathological studies have associated the presentation and progression of pathological conditions with changes in the fatty acyl moieties of CLs, as seen in Barth syndrome, an X-linked disease, where a change in the fatty acid composition of CL is detected [10-14]. The onset of heart failure in spontaneous hypertensive rats was also linked to the shifting of fatty acids in myocardial CL from saturated C18 fatty acids to highly unsaturated C20 and C22 species [14]. These examples highlight the biological and pathological importance of CL.

Conventional mass spectrometric analysis of CL in tissue uses the extraction methods developed by Folch et al. [15] or Bligh and Dyer [16] as the starting point. The tissue is homogenized in a chloroform-methanol mixture, and the homogenate is subjected to various extraction steps followed by laborious chromatographic separation and purification before mass spectrometric analysis [14]. Such sample handling could provide detailed information on tissue lipid composition. However, the procedures consume substantial amounts of time and sample. Further, multistep sample extraction and processing could potentially cause significant amounts of sample loss and renders such approach unsuitable for the analysis of trace amounts of the molecules of interest. In this study, we report an alternative approach for direct detection of CLs from tissue sections without extensive sample processing. Upon establishing suitable experimental parameters, we surveyed the major $\mathrm{CL}$ species from rat organs tissue sections containing high concentrations of mitochondria, such as heart, liver, kidney, muscle, and testis.

\section{Materials and Methods}

\section{Animals}

All animal use and handling were approved by the Animal Care and Use Committee (ACUC) in NIDAIRP, NIH. Male Sprague-Dawley rats between 280 and $400 \mathrm{~g}$ were used in this study. Rats were euthanized by overdose isoflurane inhalation. Upon cessation of respiration, the animals were rapidly decapitated and dissected to harvest the liver, heart, kidney, testes, and a small piece of muscle (m. rectus femoris). The harvested organs were briefly washed in ice-cold normal saline three times and trimmed into tissue cubes. The tissue cubes were blot-dried and rapidly frozen in isopentane prechilled on dry ice, then wrapped in aluminum foil and stored at below $-80^{\circ} \mathrm{C}$ until sectioning. 
The frozen tissue cubes were cut into 16 or18 $\mu \mathrm{m}$ cryosections with a cryostat (CM 3050S; Leica Microsystems, Nussloch GmbH, Nussloch, Germany). The detailed sectioning and sections handling methodology was done as previously reported [17]. The sections were collected onto stainless steel MALDI targets and briefly thaw-mounted before storage at $-20^{\circ} \mathrm{C}$. Small droplets of matrix solution ( 0.1 to $0.2 \mu \mathrm{L}$ ) were manually deposited onto the tissue regions of interest shortly before MALDI-MS analyses.

\section{Matrices}

Several conventional MALDI matrices were tested; 2,6dihydroxyacetophenone (DHA; Fluka, Buchs, Switzerland), 2,5-dihydroxy benzoic acid (DHB; Aldrich, Milwaukee, WI), 2, 4, 6-trihydroxy acetophenone (THA; Fluka, Buchs, Switzerland), $\alpha$-cyano-4-hydroxycinnamic acid (CHCA; Sigma-Aldrich, Gillingham-Dorset, UK), and sinapinic acid (SA; Aldrich, Milwaukee, WI). The matrices concentrations were $30 \mathrm{mg} / \mathrm{mL}$ in $50 \%$ ethanol. In a separate series of studies, DHA was prepared in $100 \mathrm{mM}$ cesium iodide solution (Sigma Chemical Co.; St. Louis, MO) at a $30 \mathrm{mg} / \mathrm{mL}$ concentration (see below).

\section{Standard}

Bovine CL standard containing $87 \%$ of $(18: 2)$ and $8 \%$ of (18:1) fatty acyl substituents was purchased from Avanti Polar Lipids (Alabaster, AL; product number: 840012) and reconstituted in chloroform at a concentration of $10 \mathrm{mg} / \mathrm{mL}$. Equal volumes of CL standard and matrix solution were premixed before spotting onto the MALDI target. The MALDI-MS and MALDI-MS/MS spectra from (18:2) ${ }_{4}$ CL standard served as calibrants for the in situ analysis and characterization of CLs.

\section{Mass Spectrometry}

The tissue sections were analyzed with a MALDI-TOF/ TOF mass spectrometer (4700 Proteomic Analyzer; Applied Biosystems Inc., Farmingham, MA) equipped with a Nd:YAG laser (355 nm) triggered at $200 \mathrm{~Hz}$ repetition rate. The spectra were acquired in reflectron negative ion mode. The identity of the CL peaks was confirmed by MALDI-MS/MS. Each MALDI-MS spectrum consisted of the sum of 400 consecutive laser shots, while each MALDI-MS/MS spectrum consisted of the sum of 1000 consecutive laser shots.

\section{Fragments Assignment}

The fragments assignment for the MS/MS studies followed the convention of Hsu and Turk [18, 19] with a slight modification. While the $\mathrm{a} / \mathrm{b}$ ion represents the phosphatidic acid of cardiolipin (Structure 2); a $+56 / b$ +56 and $a+136 / b+136$ ions represent the intermediate fragments containing the modified central glyc- erol backbone (Structure 3) and the modified phosphoglycerol backbone (Structure 4), respectively. The presence of cesium in the fragments, if detected, was specified.

\section{Results and Discussion}

\section{Matrix Suitability}

Several conventional MALDI matrices were evaluated on myocardial tissue sections and on CL standard. The results indicated that, among the matrices tested, DHA generated the highest abundance of CL ions from both tissue and CL standard and the least interference in the 1000 to $2200 \mathrm{~m} / \mathrm{z}$ range (Figure $1 \mathrm{la}$ ). When tested ${ }^{\circ}$ on other organ sections, DHA also gave spectra of equally high quality. Nonetheless, an empirical observation indicated that DHA sublimates in high vacuum (e.g., $1 \times$ $10^{-6}$ torr or lower) within $30 \mathrm{~min}$. Therefore, all the MS and MS/MS studies were conducted as soon as practically possible after the target plates were inserted into the source chamber.

Figure 1a shows a typical MS spectrum of $(18: 2)_{4} \mathrm{CL}$ from a myocardial section of a rat ventricle. The inlet magnifies the region between $\mathrm{m} / \mathrm{z} 1420$ and $\mathrm{m} / \mathrm{z} 1520$ that contains the CL molecular ions $\left([\mathrm{M}-\mathrm{H}]^{-}\right)$at $m / z$ 1447.89, the CL-sodium adduct at $m / z$ 1469.78, and the CL-potassium adduct at $\mathrm{m} / \mathrm{z} 1485.78$. Note that the 1000 to $2200 \mathrm{~m} / \mathrm{z}$ range is relatively free of other ion species.

We also tested the effect of exogenous alkali metal ions on detection of CL under the same conditions used in Figure 1a. Figure $1 \mathrm{~b}$ shows the spectrum acquired from the section adjacent to the one used in Figure 1a that was spotted with DHA containing $100 \mathrm{mM}$ CsI to generate in situ CL-cesium adducts (CL-Cs). Including $\mathrm{Cs}^{+}$in the matrix decreases the relative abundance of endogenous sodiated and potassiated CL adducts by $\sim 50 \%$ (cf. Figure 1a), and results in the CL-Cs adduct at $\mathrm{m} / \mathrm{z} 1579.77$ becoming the base peak. High concentration of exogenous $\mathrm{Cs}^{+}$displaced the sodium and potassium adducted to CL and replaced them with CL-Cs. The exogenous $\mathrm{Cs}^{+}$might also form CL-Cs adducts with CL molecular ions. The high concentration of $\mathrm{Cs}^{+}$ additively increases the in situ molecular abundance of CL. The exogenous $\mathrm{Cs}^{+}$also shifted CL in the form of $C L-C s$ to a $m / z$ range that was relatively free of interference from other ions [20]. Such mass shifting allowed the use of a wider precursor window, thus increasing the number of precursor ions for MS/MS studies, resulting in better MS/MS data. In addition, MS/MS studies of CL-Cs adduct appear to yield more structural information than those of the CL molecular ions (see below).

To confirm the identities of $(18: 2)_{4} \mathrm{CL}(\mathrm{m} / \mathrm{z}$ 1447.89) and (18:2) ${ }_{4}$ CL-Cs $(m / z$ 1579.77) from heart muscle, these molecular ions were selected for MS/MS fragmentation. The results were compared with the MS/MS spectra from $(18: 2)_{4}$ CL and (18:2) $)_{4}$ CL-Cs standards. After a number of MS/MS studies, we observed that it 

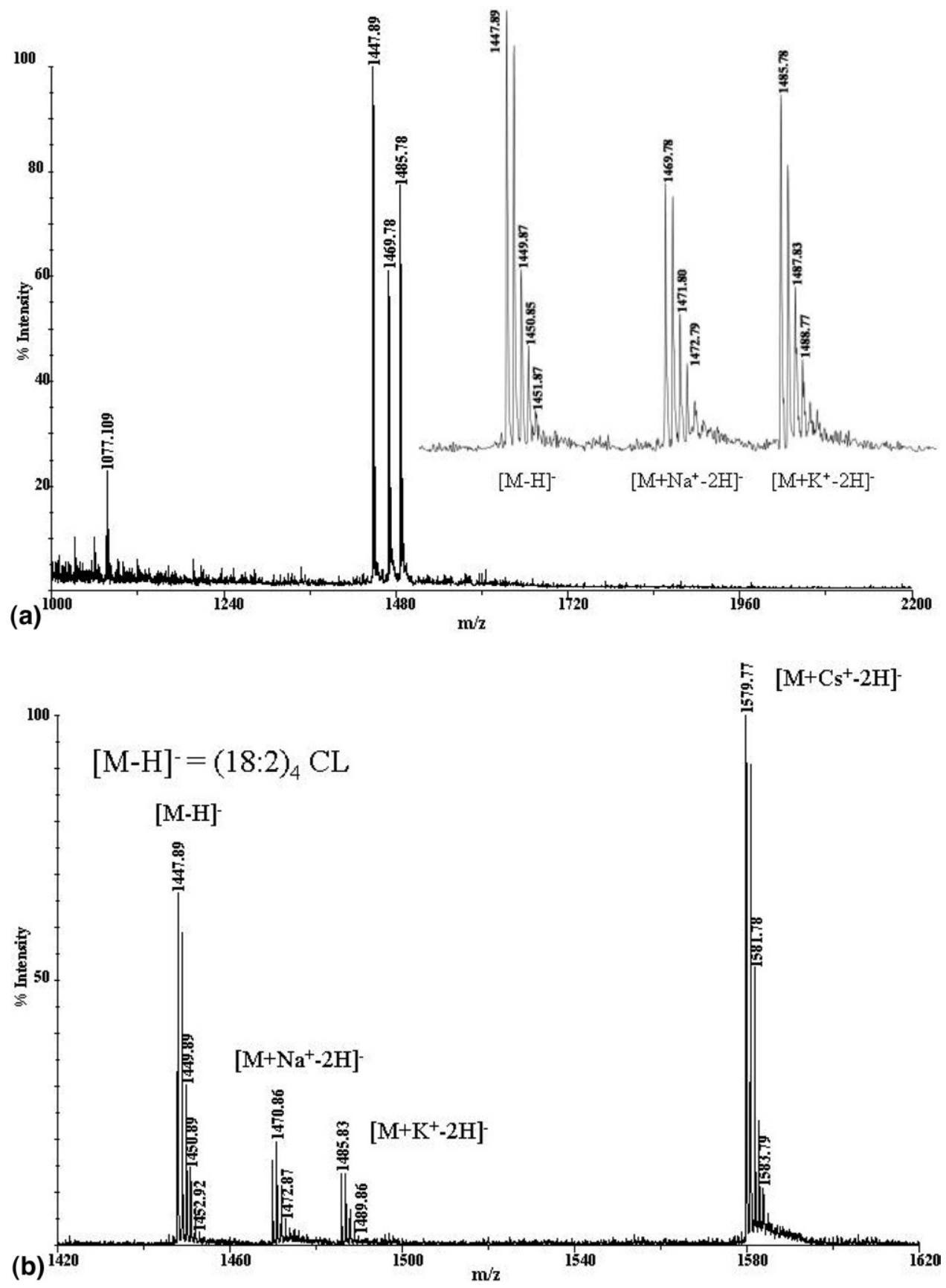

Figure 1. (a) MALDI-MS spectrum of $(18: 2)_{4}$ cardiolipin (CL) from rat heart section. The inlet magnifies the $\mathrm{m} / \mathrm{z}$ region between 1420 and $1520 \mathrm{u}$. Matrix: DHA, $30 \mathrm{mg} / \mathrm{mL}$. (b) MALDI-MS spectrum of $(18: 2){ }_{4} \mathrm{CL}$ from rat heart section in the presence of $\mathrm{Cs}^{+}$ions. The relative abundance of sodiated and potassiated CL was significantly decreased by $\mathrm{Cs}^{+}$addition.

was almost impossible to acquire structurally meaningful spectra from tissue or standards using even the lowest available collision gas (air) pressure setting $(2 \times$ $10^{-7}$ torr). All the MS/MS results showed [a-(18:2) ${ }^{-} /$ $[\mathrm{b}-(18: 2)]^{-}$ions and the (18:2) fatty acid ions, and other low $\mathrm{m} / \mathrm{z}$ peaks that are signatures of phospholipids (e.g., $\mathrm{m} / \mathrm{z}$ 153.00). Once the collision gas was turned off, the MS/MS spectra began to reveal more structurally meaningful fragments such as [a-(18:2) $]^{-} /[\mathrm{b}-(18: 2)]^{-}$ ions, $[a]^{-} /[b]^{-}$ions, $[a+56]^{-} /[b+56]^{-}$ions, $[a+136]^{-} /$ $[\mathrm{b}+136]^{-}$ions, $[\mathrm{M}-(18: 2 \text { fatty acid })-\mathrm{H}]^{-}$ion, and $\left[\mathrm{M}+\mathrm{Cs}^{+}-(18: 2 \text { fatty acid ketene)- } 2 \mathrm{H}]^{-}\right.$ions. To extract more structural information, MS/MS analysis for the remainder of the study were carried out without collision gas. Figure $2 \mathrm{a}$ shows the MS/MS spectrum of $(18: 2)_{4}$ CL molecular ion and Figure 2c the MS/MS spectrum of (18:2) ${ }_{4}$ CL-Cs adduct, both from a myocardial section. Figure $2 b$ shows the MS/MS spectrum of $(18: 2)_{4}$ CL molecular ion from standard and Figure $2 \mathrm{~d}$ the MS/MS spectrum of $(18: 2)_{4}$ CL-Cs adduct from 

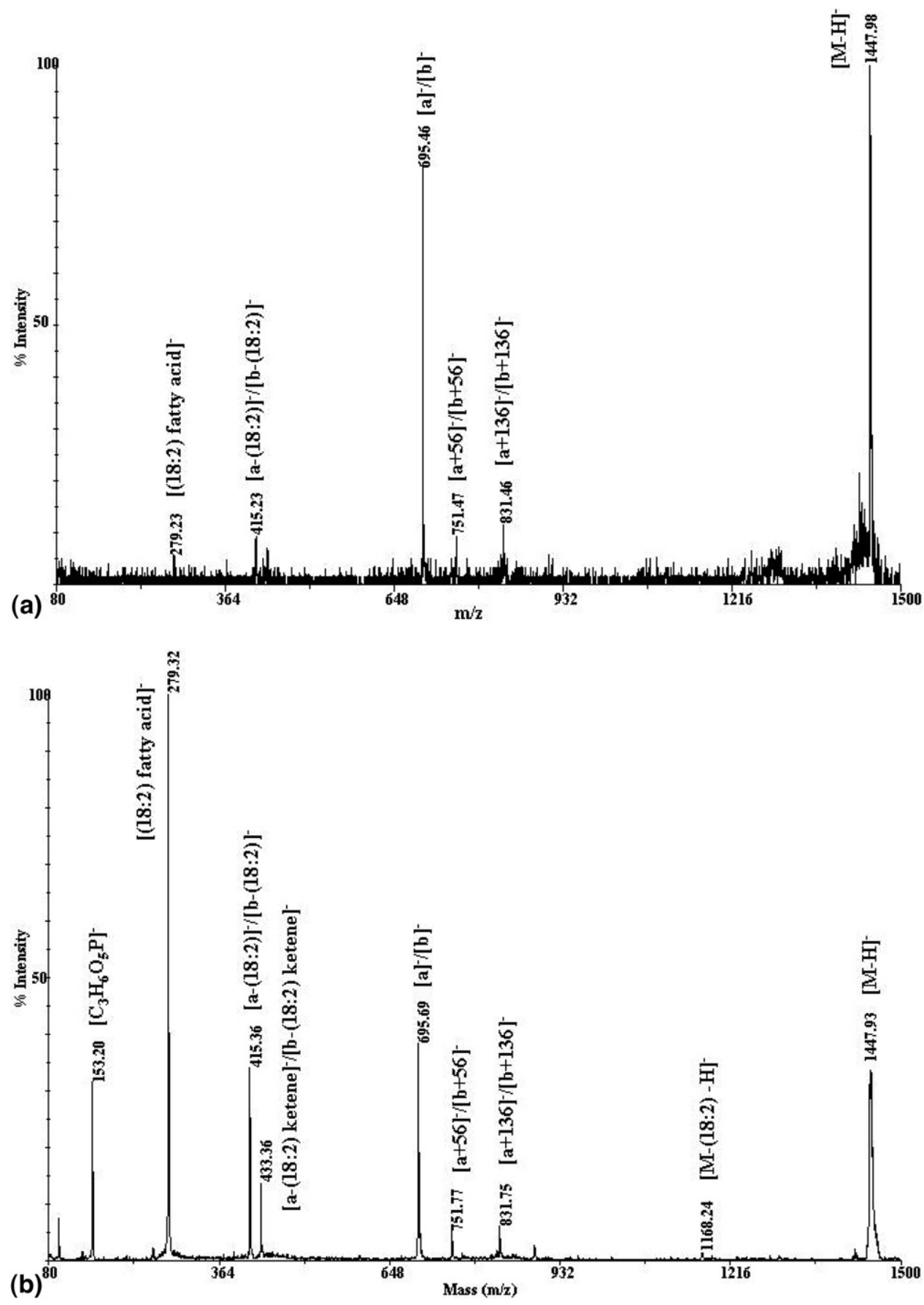

Figure 2. (a) MALDI-MS/MS spectrum of $(18: 2)_{4} \mathrm{CL}$ molecular ion $\left([\mathrm{M}-\mathrm{H}]^{-}\right)$from a rat heart section. $.^{\circ}(\mathbf{b})^{\circ} \mathrm{MALDI}-\mathrm{MS} / \mathrm{MS}^{\circ}$ spectrum $^{\circ}$ of $^{\circ}(18: 2)_{4} \mathrm{CL}$ molecular ion from CL standard.

standard. The MS/MS of CL molecular ion generated mostly $[\mathrm{a}]^{-} /[\mathrm{b}]^{-}$ions, i.e., (18:2) $)_{2}$ phosphatidic acid $(\mathrm{m} / \mathrm{z}$ 695.46), and low abundance of the following fragments: $[\mathrm{a}+56]^{-} /[\mathrm{b}+56]^{-}$ions $(\mathrm{m} / \mathrm{z} 751.47) ;[\mathrm{a}+136]^{-} /$ $[\mathrm{b}+136]^{-}$ions $\left(m / z\right.$ 831.46); $[\mathrm{a}-(18: 2)]^{-} /[\mathrm{b}-(18: 2)]^{-}$ions $(\mathrm{m} / \mathrm{z} 415.23)^{\circ}\left[18,{ }^{\circ} 19\right]^{\circ}{ }^{\circ}$ and $^{\circ}(18: 2)^{\circ}$ fatty $^{\circ}$ acid $^{\circ}$ ions $^{\circ}(\mathrm{m} / \mathrm{z}$ $279.23)^{\circ}\left(\right.$ Figure $\left.^{\circ} 2 \mathrm{a}\right) .^{\circ}{ }^{\circ}$ The $^{\circ}$ results $^{\circ}$ are $^{\circ}$ generally ${ }^{\circ}$ in $^{\circ}$ agreement with MS/MS results of CL standard shown in Figure 2b. Nonetheless, a minor peak representing

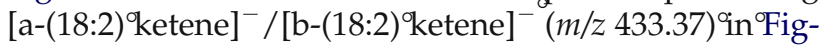

ure $2 \mathrm{~b}^{\circ}$ was not ${ }^{\circ}$ detected in the heart ${ }^{\circ}$ muscle section. The difference probably is due to the low abundance of precursor ions available in the myocardial sections.

The MS/MS of CL-Cs adduct showed a very different 9 fragmentation profile(Figure $2 \mathrm{c}$ ). $.^{\text {The }}[\mathrm{a}]^{-} /[\mathrm{b}]^{-}$ions were barely detectable, and the abundance of $[a+56]^{-} /$ $[b+56]^{-}$ions was low. However, the relative abundance of $\left[\mathrm{a}+136+\mathrm{Cs}^{+}\right]^{-} /\left[\mathrm{b}+136+\mathrm{Cs}^{+}\right]^{-}(\mathrm{m} / \mathrm{z}$ 963.37) was much higher than that of $[\mathrm{a}]^{-} /[\mathrm{b}]^{-}$and $[\mathrm{a}+56]^{-} /$ $[b+56]^{-}$ions. In addition, the spectrum also showed 

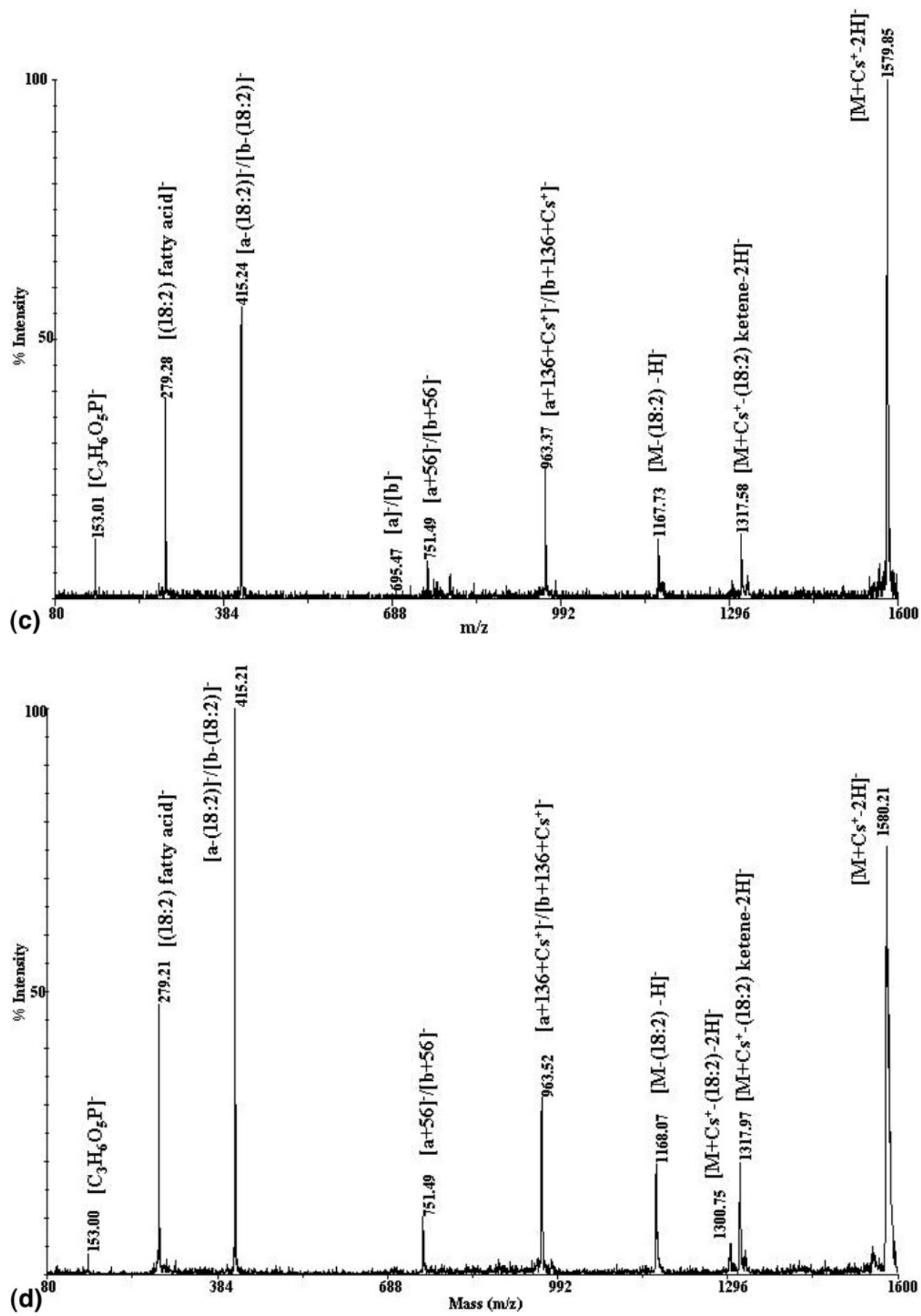

Figure 2. Continued. (c) MALDI-MS/MS spectrum of $(18: 2)_{4} \mathrm{CL}-\mathrm{Cs}$ ion $\left(\left[\mathrm{M}+\mathrm{Cs}^{+}-2 \mathrm{H}\right]^{-}\right)$from a rat heart section. (d) MALDI-MS/MS spectrum of $(18: 2){ }_{4} \mathrm{CL}-\mathrm{Cs}$ ion $\left(\left[\mathrm{M}+\mathrm{Cs}^{+}-2 \mathrm{H}\right]^{-}\right)$from $\mathrm{CL}$ standard.

moderate abundance of $[\mathrm{M}-(18: 2)-\mathrm{H}]^{-}$and $\left[\mathrm{M}+\mathrm{Cs}^{+}-\right.$ (18:2 ketene)-2H $]^{-}$species $(m / z 1167.73$ and $m / z$ 1317.58, respectively). The carboxylate or ketene loss of the $\mathrm{CL}$ precursor was not observed in the MS/MS of myocardial sections, nor were the $\left[\mathrm{a}+\mathrm{Cs}^{+}\right]^{-} /\left[\mathrm{b}+\mathrm{Cs}^{+}\right]^{-}$ions $(\mathrm{m} / \mathrm{z}$ 827.36) detected. In addition, the relative abundances of $[\mathrm{a}-(18: 2)]^{-} /[\mathrm{b}-(18: 2)]^{-}(\mathrm{m} / \mathrm{z} 415.24)$ and 18:2 fatty acid molecular ions $(m / z 279.28)$ were dramatically increased (cf. Figure 2a). The overall result in Figure 2c is largely in agreement with the MS/MS results of
CL-Cs ${ }^{\circ}$ standard ${ }^{\circ}$ in $^{\circ}$ Figure $^{\circ} 2 \mathrm{~d}^{\circ}{ }^{\circ}$ Table $^{\circ} 1^{\circ}$ summarizes $^{\circ}$ the fragments assignment of (18:2) ${ }_{4} \mathrm{CL}$ and (18:2) ${ }_{4} \mathrm{CL}-\mathrm{Cs}$.

\section{Distribution of Major CL Species in Rat Organs}

Heart. Heart muscle is a major source of CLs. A MALDI-MS $^{\circ}$ spectrum $^{\circ}\left(\text { Figure }^{\circ} 1 \mathrm{a}^{\circ}\right)^{\circ}$ shows $^{\circ}$ the ${ }^{\circ}$ molecular ion of (18:2) ${ }_{4}$ CL ( $m / z$ 1447.96), its sodiated $(m / z$ 1470.96) and its potassiated $(\mathrm{m} / \mathrm{z} 1487.24)$ adducts from the rat myocardial sections. 


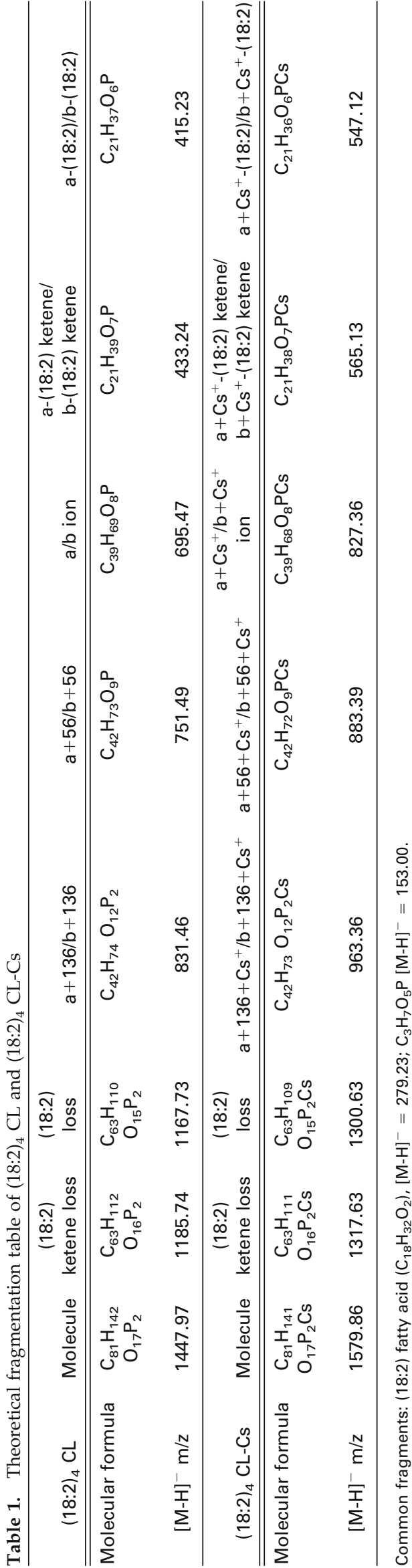

Liver. Figure 3a shows CL-Cs MS profile from a rat liver section. The majority of the CL species in liver appeared to be $(18: 2)_{4}$ CL. However, the deviation of regular isotopic distribution of CL at $\mathrm{m} / \mathrm{z} 1447.96$ cluster and that of CL-Cs cluster at $\mathrm{m} / \mathrm{z} 1579.78$ suggested the presence of a minor $(18: 2)_{3}(18: 1) \mathrm{CL}$

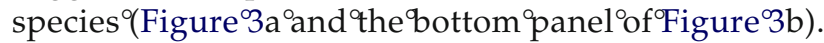
The $^{\circ}$ theoretical ${ }^{\circ}$ isotopic ${ }^{\circ}$ calculation for $(18: 2)_{4}$ CL indicated that the third isotopic peak at $\mathrm{m} / \mathrm{z} 1449.97$ should be $\sim 50 \%$ as abundant as the $\mathrm{C} 12$ peak at $\mathrm{m} / \mathrm{z}$ 1447.96. However, in Figure 3a the relative abundance of $\mathrm{m} / \mathrm{z} 1449.97$ peak was at least $90 \%$ that of $\mathrm{m} / \mathrm{z}$ 1447.97. This isotopic deviation was further emphasized by the concentrating effect of $\mathrm{Cs}^{+}$seen in the CL-Cs adduct at $m / z$ 1579.78.

Figure $3 \mathrm{~b}$ shows a more detailed isotopic comparison of the $\mathrm{m} / \mathrm{z} 1579.78$ cluster. The top panel, showing the isotopic distribution of $(18: 2)_{4}$ CL-Cs from rat heart, agrees with the theoretical calculation. The bottom panel, from rat liver, shows an increase in relative abundance of the following isotopic peak at $\mathrm{m} / \mathrm{z} 1581.78,1582.78,1583.77$, and 1584.77. The $2 \mathrm{u}$ increase is most likely due to the difference of a double-bond in one of the four fatty acid chains. The MS/MS of liver CL-Cs $(\mathrm{m} / \mathrm{z}$ 1579.78) in Figure 3c shows a very similar fragment pattern to that of $(18: 2)_{4}$ CL-Cs in Figure 2b. Nonetheless, the MS/MS also shows a $\mathrm{m} / \mathrm{z} 281.23$ peak corresponding to (18:1) fatty acid fragment, which confirms the presence of (18:1) fatty acid in liver CL. Theoretically, the $\left[\mathrm{a}+136+\mathrm{Cs}^{+}\right]^{-} /\left[\mathrm{b}+136+\mathrm{Cs}^{+}\right]^{-}$fragments and other high mass fragments should also reflect the presence of (18:1) fatty acid in liver CL. However, the spectrum did not show such a variation. This discrepancy could be due to low precursor ion abundance in spite of the efforts to increase the precursor ion counts. Also, the lack of evidence for the presence of (18:1) fatty acid chain in the $[\mathrm{M} \text {-fatty acid- } \mathrm{H}]^{-}$and $\left[\mathrm{M}+\mathrm{Cs}^{+}\right.$-fatty acid ketene-2H] $]^{-}$fragments could be due to the stereospecific numbering $(s n)$ position of the (18:1) fatty acid on CL $[18,19,21]$. According to an earlier study, the (18:1) fatty acid chain of rat liver CL was located in $s n-2$ (or $s n-2^{\prime}$ ) position [13]. In addition, according to earlier studies by Hsu and Turk [18, 19], the MS/MS of sodiated CL $\left[\mathrm{M}+\mathrm{Na}^{+}-2 \mathrm{H}\right]^{-}$preferentially dissociated the fatty acid at $s n-2$ (or $\left.s n-2^{\prime}\right)$ position.

Kidney. The MS profile of kidney CL is identical to that of liver. The major CL specie is the $(18: 2)_{4} \mathrm{CL}$, and the minor CL specie is the $(18: 2)_{3} /(18: 1) \mathrm{CL}$. Their structure was confirmed by MS/MS of CL-Cs adducts.

Leg muscle. The MS profile of CL in leg muscle appeared identical to that of heart muscle where only $(18: 2)_{4}$ CL was detected. The identity was also confirmed by MS/MS of the CL-Cs adduct. 

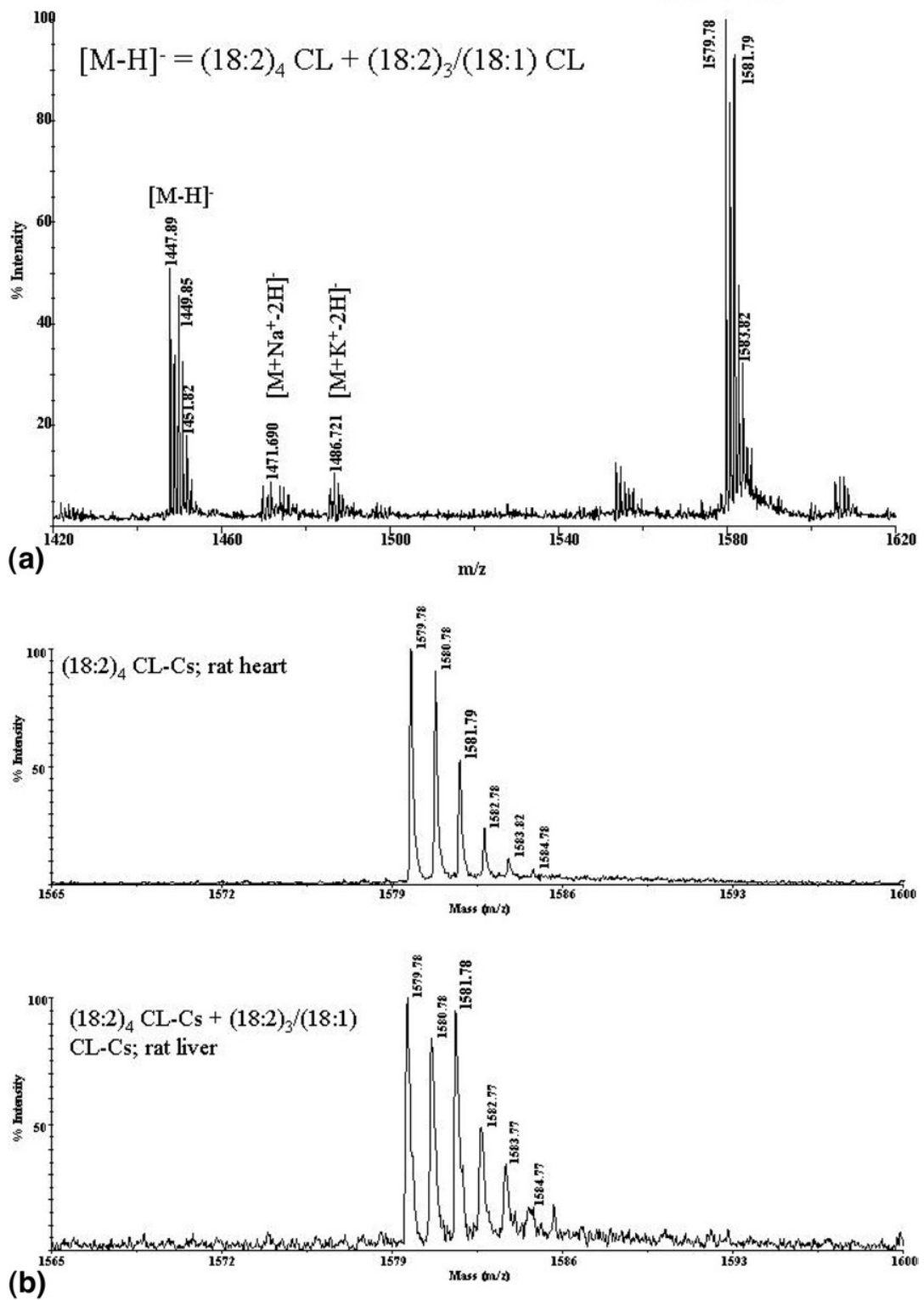

(b)

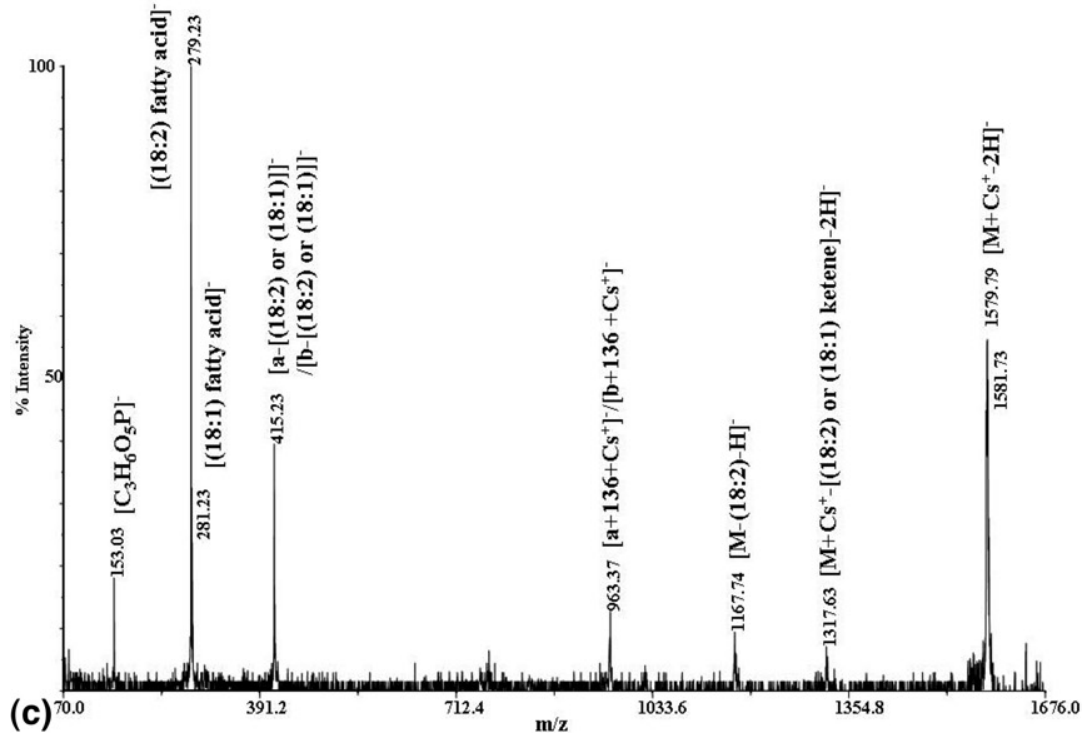

Figure 3. (a) MALDI-MS spectrum of $(18: 2)_{4}$ and $(18: 2)_{3} /(18: 1)$ CL from a rat liver section in the presence of $\mathrm{Cs}^{+}$ion. (b) Isotopic distribution of CL-Cs from a rat heart section (top panel) and a rat liver section (bottom panel). Note the increase in abundance of $\mathrm{m} / z 1581.78$ peak in the bottom panel. (c) MALDI-MS/MS spectrum of $(18: 2)_{4}+(18: 2)_{3} /(18: 1)$ CL-Cs from a rat liver section. 

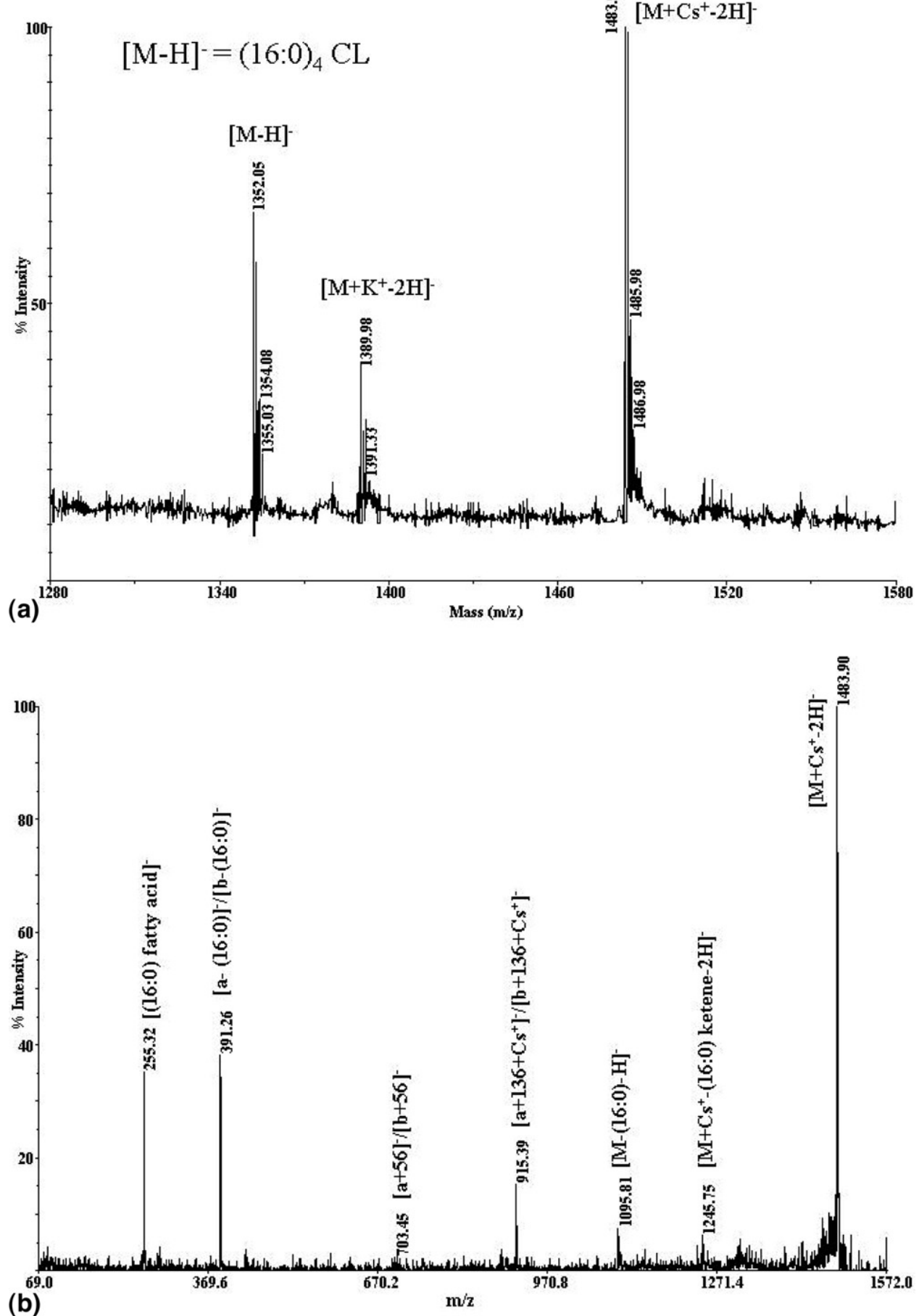

(b)

Figure 4. (a) MALDI-MS spectrum of $(16: 0)_{4}$ CL-Cs spectrum from a rat testis section, matrix containing $100 \mathrm{mM} \mathrm{CsCl}$. (b) MALDI-MS/MS spectrum of $(16: 0)_{4} \mathrm{CL}-\mathrm{Cs}\left(\left[\mathrm{M}+\mathrm{Cs}^{+}-2 \mathrm{H}\right]^{-}\right)$from a rat testis section.

Testis. Figure 4a shows the MS profile of CL from testis. Unlike the CL profiles in other organs, the major CL specie in testis is the $(16: 0)_{4} \mathrm{CL}$ at $\mathrm{m} / \mathrm{z} 1352.05$, whose cesium adduct is at $\mathrm{m} / \mathrm{z} 1483.94$. Figure $4 \mathrm{~b}$ shows the $\mathrm{MS} / \mathrm{MS}^{\circ}$ spectrum $^{\circ}$ of $^{\circ}(16: 0)^{\circ} \mathrm{CL}^{-\mathrm{C}} .^{\circ}{ }^{\circ}$ Table $^{\circ} 2^{\circ}$ lists $^{\circ}$ the fragments of $(16: 0)_{4} \mathrm{CL}$ and $(16: 0)_{4}$ CL-Cs. The $\mathrm{m} / \mathrm{z}$ 1245.75 peak represents the $(16: 0)_{4}$ CL-Cs losing one fatty acid as a ketene, while the $m / z 1095.81$ peak represents the loss of a fatty acid and $\mathrm{Cs}^{+}$from CL-Cs adduct. The $\mathrm{m} / \mathrm{z} 915.39$ peak represents the $\left[\mathrm{a}+136+\mathrm{Cs}^{+}\right]^{-} /\left[\mathrm{b}+136+\mathrm{Cs}^{+}\right]^{-}$fragment. The relative abundance of $[a+56]^{-} /[b+56]^{-}$ions was again quite low, and the $[\mathrm{a}]^{-} /[\mathrm{b}]^{-}$ions were essentially undetectable. A $m / z 391.26$ peak, representing [a-(16:0) $]^{-} /[\mathrm{b}-(16$ : 


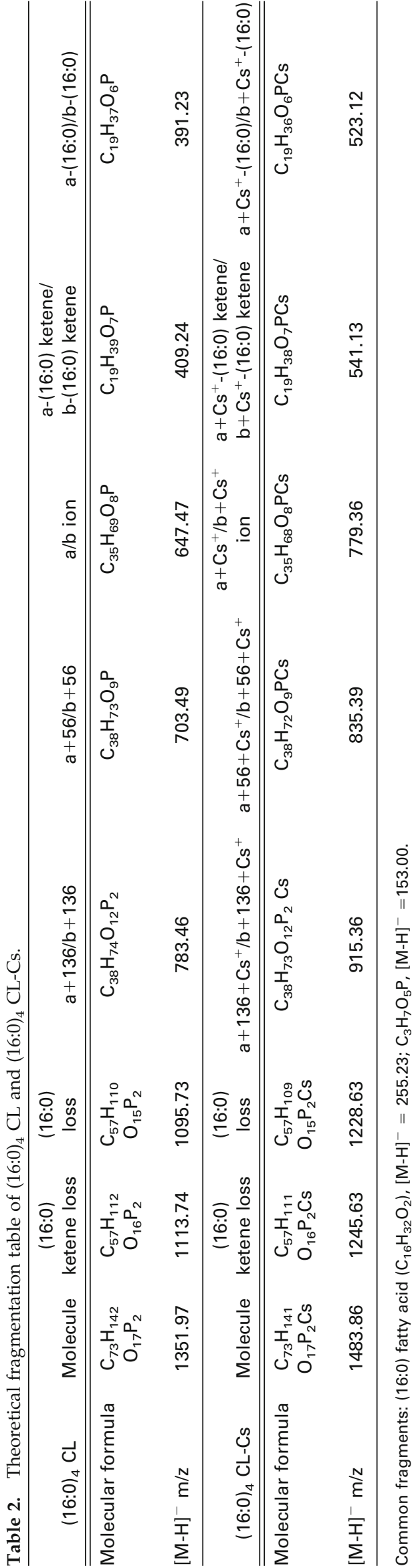

$0)]^{-}$fragment, and the (16:0) fatty acid ion at $\mathrm{m} / \mathrm{z} 255.32$ were also detected. Overall, the MS/MS profile of $(16: 0)_{4}$ CL-Cs is very similar to that of (18:2) CL-Cs seen in Figure 2c.

In this study, we demonstrated that proper selection of MALDI matrix and sample preparation are critical for the success of direct analysis of lipids in tissue sections. In our experience, DHA is the most suitable matrix for such applications. When the relative abundance of the molecules of interest is low, matrix selection and instrument tuning will influence significantly the outcome of the studies. In spite of being the preferred matrix for lipid detection, the sublimation of DHA in high vacuum within 30 to $45 \mathrm{~min}$ creates a minor inconvenience that can be overcome by respotting the matrix solution onto the regions defined by the prior matrix deposition.

It is interesting to note that MS/MS of CL molecular ion generated a fatty acid loss as ketene, which was presented as [a-(fatty acid ketene)/b-(fatty acid ketene) $]^{-}$ ion $^{\circ}$ (Figure ${ }^{\circ} 2 \mathrm{~b}$ ), ${ }^{\circ}$ while ${ }^{\circ}$ it $^{\circ}$ is ${ }^{\circ}$ presented ${ }^{\circ}$ as $^{\circ}\left[\mathrm{M}+\mathrm{Cs}^{+}\right.$-(fatty acid ketene)-2H] ${ }^{-}$ion when CL-Cs was the precursor (Figure 2d). This ${ }^{\circ}$ phenomenon ${ }^{\circ}$ was $^{\circ}{ }^{\circ}$ onfirmed by $^{\circ} \mathrm{MS} / \mathrm{MS}$ of CL-Na as the precursor (spectrum not shown). The alkali metal ion increases the basicity of gaseous CL ion and lead to a preferential $s n-2$ fatty acid loss as a ketene, as previously depicted $[18,19,21,22]$. The exogenous $\mathrm{Cs}^{+}$ seemed to neutralize the negative charge on one of the phosphate groups of $\mathrm{CL}$, preventing its access to the exchangeable hydrogen on the central glycerol, hence driving the fragmentation path towards a preferential formation of $\left[\mathrm{a}+136+\mathrm{Cs}^{+}\right]^{-} /\left[\mathrm{b}+136+\mathrm{Cs}^{+}\right]^{-}$ions. In the absence of $\mathrm{Cs}^{+}$, the fragmentation path was driven towards the formation of $[\mathrm{a}]^{-} /[\mathrm{b}]^{-}$ions that derived from $[a+56]^{-} /[b+56]^{-}$fragment ions $[19,22]$.

Our study showed that the predominant CL species in rat heart, leg muscle, liver, and kidney is the $(18: 2)_{4}$ CL; we also detected a minor species $(18: 2)_{3} /$ (18:1) CL in liver and kidney. The relative abundance of liver $(18: 2)_{3} /(18: 1) C L$, as indicated by the relative abundance of (18:1) fatty acid $(\mathrm{m} / \mathrm{z} 281.23)$, was about 25 to $30 \%$ of that of $(18: 2)$ fatty acid $(\mathrm{m} / \mathrm{z} 279.23)$. These results appear largely in agreement with the study by Schlame et al. on CL species distribution in mammalian organs [13].

The CL in testes, however, is dominated by $(16: 0)_{4}$ CL. Such distribution difference may be the result of evolutionary adaptation of spermatozoa to their environment. Clinical studies have associated sterility with oxidative stress-mediated DNA damage in spermatozoa [23-25]. As was previously mentioned, mitochondrial CL would dissociate from the Cyt C-CL complex upon oxidation, and subsequently trigger a cascade of apoptotic events [7-9]. Since lipid peroxidation usually occurs at the unsaturated bonds of fatty acids, elimination of unsaturated fatty acids in testicular CL could be regarded as the result of an evolutionary-derived protective mechanism to increase the fertility of spermatozoa by reducing their chance of undergoing lipid per- 
oxidation through oxidative stress in its highly specialized functional environment [23-25].

One of the advantages in MALDI-MS detection of CLs over ESI-MS technique is that, unlike in ESI where multiply charged ions are seen, the entire population of CL species in MALDI-MS are detected as singly charged ion species. Analysis of singly charged CL species allows a clearer separation of their isotopic peaks and might offer more accurate relative abundance information of molecular species that are separated by only 2 or $4 \mathrm{u}$ [14]. The detection of mixed CL species in liver by its isotopic pattern and the subsequent MS/MS confirmation of its (18:1) fatty acid relative abundance further signify the advantages of MALDI-MS in such applications.

Although MALDI-MS has the advantage of generating singly charged molecular ions over ESI-MS, the latter will always be the technique of choice for quantitative analysis [26]. In addition, due to instrumental limitation, we could only perform MS/MS on the precursor ions. Further identification of the fragment ions generated by MS/MS will require an ion trap mass spectrometer for conducting $\mathrm{MS}^{\mathrm{n}}$ fragmentation.

In summary, with proper matrix selection and instrument setting, the in situ analysis of CL from organ sections containing high concentrations of mitochondria is achievable. Further modification and refinement of the current method could permit its adaptation for the fast screening of changes in lipid biomarkers associated with mitochondria related diseases in biopsy samples, and would offer a rapid turnaround time with little sample consumption.

\section{Acknowledgments}

This research was supported by the Intramural Research Program of the National Institute on Drug Abuse, NIH. The authors thank the Office of National Drug Control Policy (ONDCP) for instrumentation funding, without which this and other projects could not have been accomplished.

\section{References}

1. Haiens, T. H.; Dencher, N. A. Cardiolipin: A Proton Trap for Oxidative Phosphorylation. FEBS Letts. 2002, 528, 35-39.

2. Pangborn, M. Isolation and purification of a serologically active phospholipid from beef heart. J. Biol. Chem. 1942, 143, 247-256.

3. Gary, G. M.; Macfarlane, M. G. Separation and Composition of the Phospholipids of Ox Heart. Biochem. J. 1958, 70, 409-425.

4. Macfarlane, M. G. Structure of Cardiolipin. Nature 1958, 182, 946.

5. Macfarlane, M. G.; Wheeldon, L. W. Position of the Fatty Acids in Cardiolipin. Nature 1959, 183, 1808.
6. Daum, G. Lipids of Mitochondria. Biochim. Biophys. Acta 1985, 822(1), $1-42$.

7. Kagan, V. E.; Borisenko, G. G.; Tyurina, Y. Y.; Tyurin, V. A.; Jiang, J.; Potapovich, A. I.; Kini, V.; Amoscato, A. A.; Fujii, Y. Oxidative Lipidomics of Apoptosis: Redox Catalytic Interactions of Cytochrome $c$ with Cardiolipin and Phosphatidylserine. Free Rad. Biol. Med. 2004, 15, 37(12), 1963-1985.

8. McMillin, J. B.; Dowhan, W. Cardiolipin and Apoptosis. Biochim. Biophys. Acta 2002, 1585(2/3), 97-107

9. Iverson, S. L.; Orrenius, S. The Cardiolipin-Cytochrome $c$ Interaction and the Mitochondrial Regulation of Apoptosis. Arch. Biochem. Biophys. 2004, 423(1), 37-46.

10. Valianpour, F.; Wanders, R. J.; Overmars, H.; Vreken, P.; Van Gennip, A. H.; Baas, F.; Plecko, B.; Santer, R.; Becker, K.; Barth, P. G. Cardiolipin Deficiency in X-Linked Cardioskeletal Myopathy and Neutropenia (Barth Syndrome, MIM 302060): A Study in Cultured Skin Fibroblasts. J. Pediatr. 2002, 141(5), 729-33.

11. Schlame, M.; Towbin, J. A.; Heerdt, P. M.; Jehle, R.; DiMauro, S.; Blanck, T. J. Deficiency of Tetralinoleoyl-Cardiolipin in Barth Syndrome. Ann. Neurol. 2002, 51(5), 634-637.

12. Schlame, M.; Kelley, R. I.; Feigenbaum, A.; Towbin, J. A.; Heerdt, P. M.; Schieble, T.; Wanders, R. J.; DiMauro, S.; Blanck, T. J. Phospholipid Abnormalities in Children with Barth Syndrome. J. Am. Coll. Cardiol. 2003, 42(11), 1994-1999.

13. Schlame, M.; Ren, M.; Xu, Y.; Greenberg, M. L.; Haller, I. Molecular Symmetry in Mitochondrial Cardiolipins. Chem. Phys. Lipids 2005, 138(1/2), 38-49.

14. Sparagna, G. C.; Johnson, C. A.; McCune, S. A.; Moore, R. L.; Murphy, R. C. Quantitation of Cardiolipin Molecular Species in Spontaneously Hypertensive Heart Failure Rats Using Electrospray Ionization Mass Spectrometry. J. Lipid Res. 2005, 46(6), 1196-1204.

15. Folch, J.; Lees, M.; Sloane Stanley, G. H. A Simple Method for the Isolation and Purification of Total Lipids from Animal Tissues. J. Biol. Chem. 1957, 226(1), 497-509.

16. Bligh, E. G.; Dyer, W. J. A Rapid Method of Total Lipid Extraction and Purification. Can. J. Biochem. Physiol. 1959, 37(8), 911-917.

17. Jackson, S. N.; Wang, H. Y.; Woods, A. S.; Ugarov, M.; Egan, T.; Schultz, J. A. Direct Tissue Analysis of Phospholipids in Rat Brain Using MALDI-TOFMS and MALDI-Ion Mobility-TOFMS. J. Am. Soc. Mass Spectrom. 2005, 16(2), 133-138.

18. Hsu, F. F.; Turk, J.; Rhoades, E. R.; Russell, D. G.; Shi, Y.; Groisman, E. A Structural Characterization of Cardiolipin by Tandem Quadrupole and Multiple-Stage Quadrupole Ion-Trap Mass Spectrometry with Electrospray Ionization. J. Am. Soc. Mass Spectrom. 2005, 16(4), 491-504.

19. Hsu, F. F.; Turk, J. Characterization of Cardiolipin from Escherichia coli by Electrospray Ionization with Multiple Stage Quadrupole Ion-Trap Mass Spectrometric analysis of $[\mathrm{M}-2 \mathrm{H}+\mathrm{Na}]^{-}$ions. J. Am. Soc. Mass Spectrom. 2006, 17(3), 420-429.

20. Schiller, J.; Suss, R.; Petkovic, M.; Hilbert, N.; Muller, M.; Zschornig, O. Arnhold, J.; Arnold, K. CsCl as an Auxiliary Reagent for the Analysis of Phosphatidylcholine Mixtures by Matrix-Assisted Laser Desorption and Ionization Time-of-Flight Mass Spectrometry (MALDI-TOF MS). Chem. Phys. Lipids 2001, 113(1/2), 123-131.

21. Hsu, F. F.; Turk, J. Studies on Phosphatidylserine by Tandem Quadrupole and Multiple Stage Quadrupole Ion-Trap Mass Spectrometry with Electrospray Ionization: Structural Characterization and the Fragmentation Processes. J. Am. Soc. Mass Spectrom. 2005, 16(9), 1510-1522.

22. Hsu, F. F.; Turk, J. Charge-Remote and Charge-Driven Fragmentation Processes in Diacyl Glycerophosphoethanolamine Upon Low-Energy Collisional Activation: A Mechanistic Proposal. J. Am. Soc. Mass Spectrom. 2000, 11(10), 892-899.

23. Sikka, S. C. Relative Impact of Oxidative Stress on Male Reproductive Function. Curr. Med. Chem. 2001, 8(7), 851-862.

24. Aitken, R. J.; Baker, M. A. Oxidative Stress and Male Reproductive Biology. Reprod. Fertil. Dev. 2004, 16(5), 581-588.

25. Agarwal, A.; Gupta, S.; Sikka, S. The Role of Free Radicals and Antioxidants in Reproduction. Curr. Opin. Obstet. Gynecol. 2006, 18(3), 325-332.

26. Han, X.; Yang, J.; Cheng, H.; Yang, K.; Abendschein, D. R.; Gross, R. W. Shotgun Lipidomics Identifies Cardiolipin Depletion in Diabetic Myocardium Linking Altered Substrate Utilization with Mitochondrial Dysfunction. Biochemistry 2005, 44(50), 16684-16694. 